(C) 1981. The Genetical Society of Great Britain

\title{
THE FREQUENCY OF HETEROZYGOTES MAINTAINED IN SYNTHETIC POPULATIONS OF NICOTIANA RUSTICA
}

HILARIE J. BOUGHEY, CLAIRE DOUGLAS, M. K. S. AL-BANNA and J. L. JINKS

Department of Genetics, University of Birmingham, Birmingham B15 2TT

Received i.v.81

\section{INTRODUCTION}

UNDER natural conditions, Nicotiana rustica sets a proportion of its seed by outcrossing, the rest by selfing. There is, however, considerable variation in the relative positions of stigma and anthers in the corolla tube (Paxman, 1956) and in the timing of anther dehiscence relative to the opening of the flower, and both influence the rate of outcrossing (Breese, 1959). Selections for stigmas that are higher than the anthers, have a mean outcrossing rate of 26.6 per cent compared with 10 per cent in selections for stigmas that are lower than the anthers.

As part of a long term investigation of equilibrium conditions in synthetic populations of $N$. rustica initiated from mixtures of pure-breeding lines, the frequency of heterozygotes has been monitored over three successive generations. This frequency appears to be increasing faster than initial estimates of the rate of outcrossing predict.

\section{MATERIALS AND METHODS}

Two contrasting synthetic populations have been investigated. The $B$ population was initiated from a random sample of 82 pure breeding lines derived by single seed descent from a cross between varieties 1 and 5 (Perkins and Jinks, 1973). The $\mathrm{D}$ population was initiated from a random sample of 60 pure breeding lines derived in the same way from a cross between varieties 2 and 12 (Jinks et al., 1977). The heritable variation in the cross of varieties 2 and 12 is approximately twice as great as that in the cross of varieties 1 and 5 and dominance and non-allelic interactions make a proportionately greater contribution (Jinks and Perkins, 1970; Pooni, Jinks and Jayasekara, 1978). Both populations are polymorphic for ovary colour, the dominant black ovary allele $(A)$ having been introduced by varieties 1 and 12 and the recessive green ovary allele $(a)$ by varieties 2 and 5 . Thirty nine of the $82 \mathrm{~B}$ lines and 27 of the $60 \mathrm{D}$ lines are homozygous $(\boldsymbol{A} \boldsymbol{A})$ for black ovary. There is no association between this marker and any other phenotypic difference among the lines.

The initial, $S_{0}$, generation of the $D$ population was set up in 1975 from 35 plants of each of the 60 lines in a completely randomised design. The $S_{0}$ generation of the $B$ population was set up a year later from 50 plants of each of the 82 lines raised at twice the density, because of their smaller size, in a completely randomised design. The populations were maintained in complete isolation at Avoncroft. Since they were protected from predation by enclosure in a net, interplant competition was the principal selective force. 
$S_{1}, S_{2}$ and $S_{3}$ generations each of 2000 completely randomised plants were raised from random samples of the seed produced by the previous generation. In 1977 only, the $S_{1}$ of the $B$ population consisted of 4000 plants to match the $S_{0}$ generation.

The progress of each population was monitored by growing a parallel random sample of the $S_{1}, S_{2}$ and $S_{3}$ generations at Edgbaston for observation, comparison with controls and breeding tests. Each of these assessments was designed to consist of 900 plants from the D and 820 plants from the $\mathrm{B}$ populations. They were completely randomised with the same number of $S_{0}$ and $F_{2}$ plants as controls which are not relevant to the present paper. A single major gene controlled difference in ovary colour, black versus green (Pooni and Jinks, 1981) was scored on all plants and a random sub-sample of those with black ovaries were selfed. Twenty plants of each of these progenies were raised in the glasshouse over winter the intention being to score at least 16 for ovary colour. If all the plants scored had black ovaries the parent was classified as homozygous black $(A A)$, if one or more had a green ovary the parent was classified as heterozygous black $(A a)$. If at least 16 were scored the probability of misclassifying a parent was less than one per cent. In the first sub-samples raised over winter (from the $S_{1}$ of the $B$ and $D$ populations) too many progenies fell below this target; they were therefore supplemented by second samples of random progenies raised in a subsequent winter. Because of the slow rate of change between the $S_{0}$ and $S_{1}$ for all of the other characteristics that were monitored in the $B$ population the $S_{2}$ was not assessed.

\section{Results}

The classification of the samples of $B$ and $D$ populations raised at the Edgbaston and of the sub-samples of black ovaried plants is summarised in table 1. The frequency of heterozygotes in the $n$th generation $\beta_{n}$ is obtained from this table as:

$$
\beta_{n}=\frac{(A A+A a)}{(A A+A a+a a)} \times \frac{(A a)^{\prime}}{(A A+A a)^{\prime}}
$$

TABLE 1

The frequency of black (AA + Aa) and green (aa) ovaries in samples drawn from the $B$ and $D$ populations and the frequency of homozygous (AA) and heterozygous (Aa) black ovaries in the progeny tested sub-samples of black ovaried plants

Population B

Generation

\begin{tabular}{cc}
\multicolumn{2}{c}{ Sample } \\
$(\boldsymbol{A A}+\boldsymbol{A} a)$ & $a a$ \\
364 & 413 \\
325 & 495
\end{tabular}

$\begin{array}{cc}\text { Sub-sample } & (\boldsymbol{A} A+\boldsymbol{A} a) \\ \boldsymbol{A} \boldsymbol{A} & \boldsymbol{A} a \\ 135 & 35 \\ 67 & 48\end{array}$

Population D

$\begin{array}{lll}\mathrm{S}_{1} & 877 & 914 \\ \mathrm{~S}_{2} & 550 & 344 \\ \mathrm{~S}_{3} & 529 & 361\end{array}$

$\begin{array}{rr}181 & 44 \\ 86 & 27 \\ 75 & 30\end{array}$


where $(A A+A a+a a)$ is the total number of plants in the sample of the $n$th generation, $(A A+A a)$ is the number of black ovaried plants in this sample, $(A A+A a)^{\prime}$ is the total number of plants in the sub-sample of black ovaried plants, and $(A a)^{\prime}$ is the number of plants in the sub-sample classified as heterozygotes. The estimates derived from the results in table 1 are given in table 2 . In both populations the frequency of heterozygotes increases from less than 10 per cent in the $S_{1}$ to over 16 per cent in the $S_{3}$. From the same data the frequency of the recessive allele $(a)$ in each generation $v_{n}$ can be estimated and hence the frequency of the dominant allele $(A)$ as $u_{n}=1-v_{n}$ (table 2).

\section{TABLE 2}

Estimates of the frequency of heterozygotes $\left(\beta_{n}\right)$, the frequency of the A allele $\left(\mathrm{u}_{\mathrm{n}}\right)$ and the rate of outcrossing $\left(1-\mathrm{f}_{n}\right)$ in the $\mathrm{n}$ th generation of the $B$ and $D$ populations

Population B

$\begin{array}{cccc}\text { Generation } & \beta_{n} & u_{n} & \left(1-f_{n}\right) \\ \mathrm{S}_{1} & 0.0965 & 0.4202 & 0.1980 \\ \mathrm{~S}_{3} & 0.1654 & 0.3136 & 0.2723 \\ \text { Pulation D } & & & \\ \mathrm{S}_{1} & 0.0958 & 0.4418 & 0.1942 \\ \mathrm{~S}_{2} & 0.1470 & 0.5417 & 0.2129 \\ \mathrm{~S}_{3} & 0.1698 & 0.5095 & 0.2204\end{array}$

If we assume that in each generation a proportion $(1-f)$ of the seed is produced by random mating and the rest $(f)$ by selfing, these proportions can be calculated from the estimates of $u, v$ and $\beta$ using the equations:

$$
\begin{aligned}
& \beta_{1}=2 u v(1-f) \\
& \beta_{2}=2 u v(1-f)+4 u^{2} v^{2} f(1-f) \\
& \beta_{3}=2 u v(1-f)+4 u^{2} v^{2} f(1-f)+8 u^{3} v^{3} f^{2}(1-f)
\end{aligned}
$$

which are special cases of the general formula for the $n$th generation

$$
\beta_{n}=\sum_{i=1}^{n}(2 u v)^{i}(f)^{i-1}(1-f) .
$$

At equilibrium ( $n$ very large)

$$
\beta=2 u v \frac{2(1-f)}{2-f}
$$

Estimates of $1-f$ are given in table 2 .

\section{Conclusions}

The outcrossing rates in table 2 have been estimated on the assumption that outcrossing is the only mechanism increasing the frequency of heterozygotes over generations. On this assumption the outcrossing rate has to increase between the $S_{1}$ and $S_{3}$ generations in both populations in order 
to explain the rate of increase of heterozygotes. This would require that the proportion of genotypes with an above average outcrossing rate was also increasing. If, however, it is assumed that the outcrossing rate is constant then some additional mechanism must be contributing to the higher than expected frequency of heterozygotes in the $S_{3}$ generation. This additional mechanism can only be that the heterozygotes themselves have an above average competitive ability. On either explanation the frequency of heterozygotes maintained in the population at equilibrium will be greater than the initial estimates of the rate of outcrossing leads us to expect. Indeed by the $S_{3}$ the observed proportions of heterozygotes in both populations have already exceeded the expected equilibrium proportion of 0.14 to $0 \cdot 16$ derived from the outcrossing rate in the $S_{1}$ (table 2 ).

\section{REFERENCES}

BREESE, E. L. 1959. Selection for differing degrees of outbreeding in Nicotiana rustica. Ann. Bot., 23, 231-344.

JINKS, J. L., JAYASEKARA, N. E. M., AND BOUGHEY, H. 1977. Joint selection for both extremes of mean performance and of sensitivity to a macro-environmental variable. II. Single seed descent. Heredity, 39, 345-355.

JINKS, J. L., AND PERKINS, J. M. 1970. A general method for the detection of additive, dominance and epistatic components of variation. III. $F_{2}$ and backcross populations. Heredity, 25, 419-429.

PAXMAN, G. J. 1956. Differentiation and stability in the development of Nicotiana rustica. Ann. Bot., 20, 331-347.

PERKINS, J. M., AND JINKS, J. L. 1973. The assessment and specificity of environmental and genotype-environmental components of variability. Heredity, 30, 111-126.

POONI, H. S., AND JINKS, J. L. 1981. Colour of floral parts in Nicotiana rustica. Heredity, 46, 273-275.

POONI, H. S., JINKS, J. L., AND JAY ASEKARA, N. E. M. 1978. An investigation of gene action and genotype $\times$ environment interaction in two crosses of Nicotiana rustica by triple test cross and inbred line analysis. Heredity, 41, 83-92. 